BMJ Open Diabetes

Research \& Care

\title{
Providing culturally sensitive diabetes self-management education and support for black African and Caribbean communities: a qualitative exploration of the challenges experienced by healthcare practitioners in inner London
}

\author{
Louise M Goff (D) , ${ }^{1}$ Amanda Moore, ${ }^{1}$ Seeromanie Harding, ${ }^{1}$ Carol Rivas ${ }^{2}$
}

To cite: Goff LM, Moore A, Harding S, et al. Providing culturally sensitive diabetes self-management education and support for black African and Caribbean communities: a qualitative exploration of the challenges experienced by healthcare practitioners in inner London. BMJ Open Diab Res Care 2020;8:e001818. doi:10.1136/ bmjdrc-2020-001818

Received 6 August 2020 Revised 29 0ctober 2020 Accepted 17 November 2020

Check for updates

\section{(C) Author(s) (or their} employer(s)) 2020. Re-use permitted under CC BY-NC. No commercial re-use. See rights and permissions. Published by BMJ.

${ }^{1}$ Department of Nutritional Sciences, King's College London, London, UK ${ }^{2}$ Department of Social Science, University College London, London, UK

Correspondence to Dr Louise M Goff; louise.goff@kcl.ac.uk

\section{ABSTRACT}

Introduction Poor access to, and engagement with, diabetes healthcare is a significant issue for black British communities who are disproportionately burdened by type 2 diabetes (T2D). Tackling these inequalities is a healthcare priority. The purpose of this research was to explore the experiences of healthcare practitioners providing diabetes selfmanagement education and support (DSMES) to African and Caribbean adults living with T2D to inform the development of a culturally tailored DSMES program. Research design and methods Semi-structured interviews were carried out with a range of healthcare practitioners including diabetes specialist nurses, dietitians and general practitioners based in primary care in inner London. Thematic content analysis was used to identify barriers and facilitators relating to the provision of effective DSMES.

Results Ten interviews were conducted. There was a strong consensus among healthcare practitioners for the importance of DSMES in T2D healthcare. However, practitioners discussed this area of practice as overwhelmingly challenging and recognized a wide range of barriers that they face. Four themes were identified: (1) The tension between structural and responsive care needs, particularly with growing numbers of patients alongside incentivized targets driving a care agenda that does not meet the needs of diverse communities; (2) challenges posed by cultural beliefs and practices, particularly a distrust of conventional medicine, rejection of body mass index standards and a belief in 'God's will'; (3) building relationships through cultural understanding: insiders and outsiders, particularly the benefits of racial concordance and cultural knowledge/resources and (4) getting the messages across, particularly the need to address gaps in structured education.

Conclusion Provision of culturally sensitive DSMES is a challenging area of practice for practitioners, who recognize the need for more training and resources to support them in developing cultural competence. Nonetheless, practitioners recognize the importance of DSMES and are striving to provide culturally sensitive care to their patients.

\section{Significance of this study}

What is already known about this subject?

- Poor access to, and engagement with, diabetes healthcare is a significant issue for black British communities who are disproportionately burdened by type 2 diabetes. Tackling these inequalities is a healthcare priority.

What are the new findings?

- Healthcare practitioners are often sensitive to the issues of, but lack confidence in, providing diabetes self-management education and support to black African and Caribbean communities.

- An institutional lack of resources and a focus on achieving incentivized targets prevent practitioners delivering care that is responsive to the needs of their local communities.

- Information provided in existing structured education courses is generic and not sensitive to the needs of ethnically diverse communities.

How might these results change the focus of research or clinical practice?

- Healthcare practitioners in clinical practice require more education, training and resources to improve their cultural competence and feel more confident in supporting black British people in developing diabetes self-management skills. Research is needed to understand whether improving the cultural appropriateness of DSMES is able to improve healthcare engagement and clinical outcomes for black British and other ethnic minority patient groups.

\section{INTRODUCTION}

Poor access to, and engagement with, diabetes healthcare is a significant issue for black, Asian and minority ethnic (BAME) groups in the UK, despite healthcare being free at the point of delivery. ${ }^{1}$ This is a particular problem for black African and Caribbean (AfC) 
communities who are disproportionately burdened by type 2 diabetes (T2D). ${ }^{2}$ Compared with white Europeans, the prevalence is estimated to be around three times higher ${ }^{3}$ and the average age of onset is around 10 years earlier. ${ }^{4}$ Tackling these inequalities is a healthcare priority. $^{5}$

Diabetes self-management education and support (DSMES) forms the cornerstone of T2D management. DSMES focuses on empowering people to take an active role in their day-to-day management, equipping them with the necessary knowledge, skills and motivation to adopt a healthy lifestyle and become competent self-managers of the daily complexities of diabetes. ${ }^{67}$ Effective DSMES takes a collaborative approach in which the person living with T2D actively collaborates with healthcare practitioners in an educational process. ${ }^{8-10}$ In the UK, DSMES is principally delivered in primary care; management guidelines recommend that all people with newly diagnosed T2D attend a structured education course to teach them the principles of self-management. ${ }^{6}$ Courses are typically delivered by diabetes specialist nurses or dietitians, using a group structure and face-to-face delivery. Despite referral to structured education courses being incentivized in primary care, BAME groups are more likely to report that they have not had the opportunity to attend an education course than white populations. ${ }^{11}$ Furthermore, among AfC communities, specific cultural barriers to healthcare engagement are recognized. ${ }^{12} 13$ Healthcare practitioners are perceived as lacking cultural understanding and their advice as being poorly adapted or lacking cultural relevance. ${ }^{14}$

Tailoring healthcare to be more culturally sensitive is proposed as a principal means by which to address ethnic inequalities in healthcare access. ${ }^{15}{ }^{16}$ Culturally tailored healthcare is also identified as a priority by patients. ${ }^{5}$ In the UK, guidelines recommend that DSMES courses should be responsive to the cultural and linguistic needs of the populations they serve, ${ }^{6}$ but to date, no culturally tailored T2D education programs have been evaluated for UK AfC communities. ${ }^{15}$ Programs tailored for African-American communities have demonstrated significant benefits to HbAlc and diabetes knowledge. Their focus is on adapting existing programs using lay community health workers as facilitators/educators and using community settings such as churches. ${ }^{17} 18$

The aim of this research was to conduct semistructured interviews with primary care healthcare practitioners involved in T2D management in inner London, to understand their perceptions of issues relating to the healthcare needs and engagement among AfC communities with T2D and to understand their experiences of delivering healthcare to these patient groups. This study formed part of a larger program of research focusing on the development of a culturally tailored DSMES program for AfC communities, using cocreation methods involving multiple stakeholders including patients, healthcare practitioners and community leaders. ${ }^{19}$

\section{METHODS}

\section{Setting}

Three inner London boroughs (Southwark, Lambeth and Brent) were selected for their high proportional representation of AfC communities, where upwards of $25 \%$ of the local populations identify as from black ethnic backgrounds. These boroughs are also recognized to have higher levels of deprivation and higher mortality rates from preventable causes compared with London and national averages.

\section{Participants}

Healthcare practitioners involved in T2D management in both primary and intermediate care, including general practitioners, primary care practice nurses, diabetes specialist nurses and diabetes specialist dietitians, were invited for interview using purposive sampling via email. At least two healthcare practitioners within each role were recruited to provide a breadth of experiences and perspectives and to enable rich data, aiming for at least eight altogether.

\section{Procedure}

In-person semi-structured interviews were carried out between July and December 2016. Interview guides were used, comprising open questions focusing on: experience of supporting AfC patients; current care provision and challenges; and perceived patient beliefs and motivations. One researcher conducted the interviews (LMG; female senior researcher in diabetes and principal investigator for the study), only the researcher and the participant were present during the interview. The interviewer had no previous relationship with the participants. Each interview started with the researcher providing a brief overview of the research and the participant providing written informed consent. The interviews were audiorecorded, transcribed verbatim and checked for accuracy by the interviewer but were not returned to the participants for comment or checking. The transcripts were anonymized and entered into NVivo V.10 qualitative software program to facilitate data management.

\section{Data analysis}

Data were analyzed using inductive thematic content analysis. ${ }^{20}$ Analysis began with data immersion, in which the lead researcher (LMG) read and re-read the transcripts. She then undertook line-by-line open coding on three transcripts and used the one sheet of paper approach ${ }^{21}$ to develop initial conceptual themes and subthemes, which were discussed with a second author (CR). These initial themes were then applied to subsequent transcripts. As the analysis continued, themes and subthemes were compared, modified, deleted, combined and developed in line with new and alternative data. The final themes and subthemes were discussed and agreed between authors (LMG, SH and CR). Twenty per cent of the data was independently coded by a second researcher and discrepancies discussed and recoded until consensus, to 


\begin{tabular}{llll}
\hline Table 1 & Participant details & & \\
\hline Participant & Role & Sex & Ethnicity \\
\hline HP1 & Primary care practice nurse (PN) & Female & White British \\
HP2 & PN & Male & White British \\
HP3 & Diabetes specialist nurse (DSN) & Female & White British \\
HP4 & DSN & Female & African Caribbean \\
HP5 & General practitioner (GP) & Female & White British \\
HP6 & GP & Male & White British \\
HP7 & Diabetes specialist dietitian (DSD) & Female & South Asian \\
HP8 & DSN & Female & African Caribbean \\
HP10 & DSD & Female & White British \\
HP11 & DSN & Female & White British
\end{tabular}

improve validity. The aim was for descriptive themes that could inform development of the planned intervention rather than more conceptual analysis.

\section{RESULTS}

Ten interviews were conducted with a range of healthcare practitioners (table 1), who were involved in delivery of primary or intermediate care diabetes services in Lambeth, Southwark and Brent. Specifically, the diabetes specialist nurses and dietitians were all currently involved in delivery of structured education, which was the 'DESMOND' program for all included boroughs. The average length of interview was $55 \mathrm{~min}$, ranging from 39 to $69 \mathrm{~min}$.

Practitioners discussed a wide range of issues that impacted on their ability to support AfC patients in becoming effective self-managers. Four themes arose from the data:

1. The tension between structural and responsive care needs.

2. Challenges posed by cultural beliefs and practices.

3. Building relationships through cultural understanding: insiders and outsiders.

4. Getting the messages across.

These themes and their subthemes are now described. An indication of how many practitioners contributed to each theme is provided, using 'majority' for more than seven practitioners, 'most' for more than half and 'some' to indicate less than half.

\section{The tension between structural and responsive care needs}

A lack of time and money and the complexities of T2D self-management were seen as affecting practitioners and patients alike. All practitioners discussed the pressures they experience working in primary care. They described a stressful, under-resourced environment where they feel they constantly need to 'do more for less', and where services are undergoing continual change/restructuring in an attempt to work more efficiently to meet demands. The increasing demands that T2D places on services, in numbers of patients and complexity of management, and the need to consider different cultural needs, were seen by all as amplifying these more general stresses. The practice nurse below provides an example of this:

The problem I have is there are too many diabetic patients for us to manage basically in a nutshell. We simply don't have the time. (HP2, PN)

Practitioners involved in management and leadership of services described targets and financial incentives/ penalties set by both local and national agencies as principle drivers of the primary care agenda, which defined service provision priorities. A 'ticking box' model of care that enables practices to maximize income through achieving incentivized targets, rather than delivering care responsive to the needs of their local communities, caused frustration among these practitioners. Targets were recognized as particularly difficult to achieve in ethnically diverse and socially deprived communities, thus putting a significant strain on practices serving such communities, as described by the general practitioner below:

I mostly get upset because I can't hit my QOF [Quality Outcomes Framework; a system for performance management and payment of primary care services], I can't get my money in my practice. We've got to say, 'You've got to do this. You've got to do the blood pressure', and so we're delivering bad care by being target driven. (HP5, GP)

The practitioners all recognized that an open, trusted practitioner-patient relationship is an essential basis for supporting self-management but was made difficult, especially in the culturally diverse local communities, because of structural demands. Many practitioners described patients expecting/seeking a paternalistic approach, being passive in their care and struggling to grasp the principles of self-management. For example, 'Some people just get frustrated and say, 'Well, just tell me what to do" (HP10, DSD). Shifting patients from this standpoint to being effective self-managers is timeconsuming- 'it's a relationship that you build over years' (HP2, PN) - and is in direct conflict with the pressure of shorter appointment times and a lack of resources to support skill development. Targets and lack of time 
pushed practitioners to initiate sensitive conversations prematurely, which was felt to jeopardize the development of trust.

Responsive care that was less target-driven was vital in the local area, which had high levels of deprivation and unemployment. The need for patients to juggle multiple employment or caregiving responsibilities was believed to be partly responsible for poor engagement, appointment attendance and loss to follow-up, according to most practitioners. As one practice nurse explained:

Many of them, it's beyond them at the moment to do anything and I don't blame them. When I hear that my lady who hasn't engaged for a year comes in and says, 'Well, actually my older son attacked my younger one in the house with a cleaver and the police were called, and now he's gone on the run. It's me that looks after them all.' You just think, this woman is astonishingly amazing. Why on earth would she bother about her diabetes? (HP1, PN)

The combination of this perception of local needs and the demands of target-driven under-resourced care might be expected to lead to disengagement and frustration by practitioners, assuming lost to follow-up meant permanent loss of engagement, though this was suggested by only a small number of the practitioners interviewed.

\section{Challenges posed by cultural beliefs and practices}

Intersecting with the above theme, all practitioners discussed a wide range of cultural beliefs and practices, specific to their local AfC communities, that they considered to act as barriers to engaging with, and trusting, the self-management advice they were advocating.

\section{They want to treat things naturally}

AfC patient's rejection of conventional medicine and a desire for natural remedies were reported by the majority of practitioners. Patients were understood to fear insulin, associating it with death as a result of family/community experiences. Prevailing beliefs in food myths, particularly focused around supposed health promoting properties, were also discussed by most practitioners, particularly the nurses and dietitians:

The fear of going onto insulin, that people think that they're perhaps much more likely to die if they go onto insulin because they've seen family members go onto insulin very late on and then they've died from the complications. (HP11, DSN)

There's really quite a strong perception that you could heal diabetes naturally. All you need to do is find the correct diet.... so often there's problems with medication and maybe if people need to take more medication, it's quite difficult to kind of get them on board with that. (HP10, DSD)

\section{The impact of the patient's local informal networks}

The patients' local networks could be both constraining-through the influence of community perceptions and social identities-and supportive in relation to their diabetes diagnosis and its management. In both cases, this could present a barrier to self-management support from the practitioners.

The way the patient's social identity was constructed within the community was perceived by most participants to be highly influential in shaping the patient's response to diabetes and its self-management. A cultural belief in the desirability of larger body sizes, particularly among women, was recognized as a significant barrier to weight loss by the majority of participants. As one practitioner said, for example:

I have had women - particularly women - say to me, 'My

husband won't want me to lose weight'. (HP10, DSD)

This led to cultural rejection by patients of body mass index (BMI) as an assessment of body weight, as discussed by all practitioners, since eurocentric BMI measures did not account for different builds.

In addition, stigmatization could result from community perceptions of the person's health. Several practitioners, particularly nurses and dietitians, saw patients often worrying that weight loss would be misperceived as HIV illness by their social networks. Diabetes was moreover recognized by most participants to be directly associated with stigma and shame, particularly among patients of direct African ancestry. Illustrative extracts are as follows:

That gets mentioned quite a lot, 'I don't want to lose too much weight because I don't want people to think that I'm unwell'. (HP2, PN)

I think there's a lot of stigma attached with it where people don't talk about it. One person described it as being worse than a diagnosis of cancer and having to accept the diagnosis. (HP11, DSN)

There is a sense of actually I don't want to be unwell so therefore I'm going to keep it to myself and not going to tell anyone and therefore they don't get the emotional support. (HP2, PN)

This could be problematic as it was felt to result in patients often being private about their diagnosis, leading to isolation without sources of social support to help in their self-management.

All practitioners reported a strong fear of diabetes, often associated with witnessing advanced complications among close family and friends, or misunderstandings about the nature of the disease:

They're often extremely frightened about what will happen to them and they need more reassurance because they've actually lived with seeing blindness and amputation. (HP1, PN)

On the contrary, once patients did disclose to their social networks, these were recognized as powerful sources of information, communication and support, often more so than the practitioners.

It's funny how it travels, you find one person at one clinic's doing it and then another person at another 
clinic, they're doing it too. It's word of mouth I think. There's an underground network... (HP3, DSN)

\section{Building relationships through cultural understanding: insiders and outsiders}

All practitioners, whether AfC (2 of the 10) or non-AfC, felt racially concordant practitioners were better able to establish trusted, open communication with their patients and understand cultural nuances. As a result, they could be more effective in supporting AfC patients, whereas the majority of non-AfC practitioners said they held back from in-depth conversations for fear of causing offense. Examples of both are provided below:

I connect a little bit because I'm from there, I do understand about the food that we're eating. (HP4, DSN) I always used to think, here's me, this little white girl standing in front of a class of 12 middle-aged, black men. They're looking at me thinking, what does she know? I was thinking, what do I know? (HP3, DSN)

Patients' belief in 'God's will' and a strong influence of religion were recognized by most practitioners. However, practitioners who had a strong faith themselves (3 of the 10) felt confident in using positive references to faith to reframe patients' beliefs and motivate self-management engagement:

Oh, the other thing is power of prayer, of course, which is very great, and I'm a Christian so I'm very happy to talk to them about that. (HP1, PN)

All non-AfC practitioners reflected on the benefits of having cultural knowledge and culturally tailored resources in helping them open up communication, establish rapport and trust with patients and demonstrate cultural respect and understanding.

When I'm doing a DESMOND (structured education course) I might say something like, 'What's the starchy food?' People will shout out potatoes and blah, blah, blah. People will sit there quietly and then I'll say, 'What about Fufu?', and they're like, 'Ah, you know Fufu?' They smile and they look at each other, she knows what we eat. (HP3, DSN)

I now obviously know the content of the resource, but I still get it out and show them because I think it demonstrates that I'm not being disrespectful. (HP1, PN)

While it was clear that non-AfC practitioners made a great effort to expand their cultural knowledge and resources, they perceived this was only superficial; it was largely developed ad hoc through experience of interacting with AfC patients and colleagues rather than through formal training or education. More training, information and culturally tailored resources were recognized as important needs.

So we will talk about things like Afro-Caribbean food, fava, yam, plantain, green banana but actually myself I've never eaten them. So my knowledge is not brilliant. Again you don't get trained. So for me training around specific ethnic groups would be helpful. (HP2, PN)

\section{Getting the messages across}

Most practitioners perceived a preference for distinct communication styles among AfC patients, particularly for simple visual messages, with medical terminology being off-putting. Furthermore, the practitioners perceived patients favoring directness and prescriptive advice, leaving practitioners feeling conflicted between the need to tailor their communication to meet the needs of their AfC patients with their own perception of maintaining their professionalism. Examples of each of these are given below:

I think the first time that he recognised that he was overweight, through seeing that coloured chart. (HP11, DSN)

Maybe we are just too professional, or we need to simplify things, we do things or say things to this particular group. (HP7, DSD)

The concerns of practitioners regarding their limited ability and capacity to support self-management were allayed by the provision of structured education courses. Courses were perceived as the place where patients' questions would be answered, and they would be provided with the information they need for self-management.

I say, 'I'm not going to tell you too much about it today because you'll be invited on the DESMOND programme (structured education course) and that's where you'll learn everything you need to know about diabetes. (HP1, $\mathrm{PN})$

However, practitioners discussed several issues with their current structured education provision. The content was perceived as focused on education without enough behavior change support and the information as too generic and not sensitive to the needs of the local ethnically diverse communities.

So DESMOND (structured education course) is a national programme. So that's purely around education rather than motivational interviewing type techniques. (HP2, PN)

Obviously, DESMOND is standard DESMOND (structured education course), so it's not particularly tailored towards any ethnicity in terms of dietary preference or culture. (HP6, GP)

\section{DISCUSSION}

This study has explored, in depth, the experiences of healthcare practitioners providing DSMES to AfC communities living with T2D in London. There was a strong consensus for the importance of DSMES and recognition that a trusted practitioner-patient partnership was key to supporting people to become successful self-managers. Racially concordant practitioners were perceived to be better able to develop a trusting relationship with patients but, for non-concordant practitioners, the use of culturally tailored resources helped them establish rapport. However, several barriers were identified, which layer on top of one another to create 
a particularly challenging area of practice. Challenges within the healthcare system included limited appointment times, incentivized targets driving the care agenda and preventing provision of individualized care, and a lack of culturally appropriate resources. Alongside these, practitioner-based limitations, such as a lack of cultural knowledge and behavior change skills, prevented them from establishing an effective relationship with patients. A wide range of patient-based barriers further challenged the partnership, for example, a distrust of conventional medicine, rejection of BMI charts and a preference for prescriptive advice and direct communication. Diabetes structured education courses were seen as a principle source of education, but practitioners raised concerns that the courses were generic and not sensitive to the needs of patients from ethnic minority cultures. Provision of culturally sensitive DSMES and cultural training for healthcare practitioners were identified as priorities by all.

All but the racially concordant practitioners felt they had limited cultural competency and did not feel confident in their ability to provide DSMES to AfC patients because of gaps in their knowledge and understanding of AfC cultures. This may be an unexpected finding: despite living and working in London, integrated into its diversity and multiculturalism and showing some cultural sensitivity, the practitioners felt constrained in offering culturally appropriate advice and care. However, it is well reported that cultural discordance may contribute to ethnic disparities in healthcare access, ${ }^{22}$ potentially relating to the quality of patient-practitioner communication. Studies have shown that, compared with white patient groups, black patients are more likely to report lower patient-practitioner communication quality and satisfaction, less information giving, partnership building, participatory decision-making and shorter visits. However, cultural concordance is a consistent predictor of better communication quality. ${ }^{23}$ Despite perceiving their cultural knowledge to be poor, many of the cultural beliefs and practices they discussed as being held by the AfC patient groups they work with align with those reported in the literature. ${ }^{12-14} 2425$ Addressing culture in the context of education has been shown to bring about benefits to diabetes self-management and glycemic control, ${ }^{26}{ }^{27}$ illustrating the importance of practitioner knowledge and our data also show that cultural confidence is important.

Practitioners described how their knowledge was developed through informal learning, for example, shadowing AfC colleagues or visiting AfC shops, rather than formal training, which they all expressed a desire for. 'Cultural competency' in healthcare has been recognized as an integral component of addressing health inequalities. ${ }^{28}$ There are many models and frameworks, which have been developed to assist in training healthcare practitioners to develop cultural competency. The models often include dimensions of knowledge, such as understanding the meaning of culture, attitudes (eg, respecting differences in cultural norms) and skills. ${ }^{29}$ Specifically relating to T2D, our practitioners recognized the many facets of culture such as health beliefs, dietary practices and communication styles, which need to be incorporated into the education and training of healthcare practitioners. They were sensitive to the need for simple messages, avoidance of medical terminology and a preference for directness. Without patient data, we cannot validate the meaningfulness of our practitioners' cultural knowledge and understanding; however, many of the beliefs and practices they discussed align closely with patient-based themes cited in the literature,${ }^{25}$ suggesting a good level of cultural sensitivity.

Social support contributes to T2D self-management outcomes $^{3031}$ and nurturing social cohesion is another strategy shown to be effective in promoting behavior change in culturally tailored interventions aimed at AfC communities. $^{32}$ For health promotion interventions, this is often through faith-based settings, as trusted community networks. ${ }^{33}$ Our practitioners recognized the powerful influence of social networks and religion/faith on patients' beliefs and behaviors, which is commonly reported in the literature ${ }^{3435}$ and has been used as a focus in many interventions in the USA. ${ }^{33}$ However, the practitioners also discussed privacy and patients not wanting to disclose their diabetes status to their social networks, reporting a stigma associated with T2D particularly among patients of African descent. Stigma has been reported in a number of physical chronic diseases, including T2D, in several African countries, ${ }^{36}$ even in the presence of strong family support, and has been shown to undermine social and medical relationships as well as self-care. ${ }^{37}$ It is not clear to what extent issues relating to privacy and disclosure are impacting on engagement and whether attendance at group education is affected by the privacy needs of patients and how to work with this alongside the need to nurture social support in DSMES.

\section{Strengths and limitations}

Our exploration of issues relating to healthcare practitioners' experiences of providing DSMES to AfC patients and issues relating to the healthcare needs and engagement of these communities was undertaken to inform the development of a culturally tailored DSMES program for AfC communities. Engaging key stakeholders, such as practitioners, in intervention development is key to ensuring that the intervention is sensitive to the needs of the healthcare system for which it is intended and practical and implementable in real-world settings. The themes identified in this qualitative work provide rich insight into the challenges faced by healthcare practitioners and a framework for our intervention development and inform the theoretical underpinning of our intervention.

We recognize several limitations within our work. We conducted our research with a small sample of practitioners serving inner London boroughs with large AfC communities; while we believe our findings are 
transferable to other areas and other AfC communities, we cannot claim our findings to be representative and practitioners serving communities in other areas or with smaller AfC representation may have different experiences. Additionally, our sample size was relatively small for a qualitative study, however, we achieved theme saturation and feel confident that we have recognized and explored relevant issues. Our interviews were intended to focus on issues relating to the provision of healthcare for AfC communities. In doing this, we worked with practitioners serving areas with 'majority-minority' communities, whereby AfC communities represent the majority ethnic group and our interview guide focused on working with AfC patient groups; in our analysis, we have made the implicit assumption that the issues discussed relate to ethnicity per se but these issues might also relate and apply to other groups who have barriers to healthcare engagement, for example, deprived communities and intersection of ethnicity with these. We chose an inductive approach in this qualitative study to understand the experiences and perceptions of practitioners. By their very nature, the themes arising from this work are subjective. Alternative methods, such as objective score card sheets, which use a more deductive approach could provide interesting findings and could be used to broaden our study to a larger population. Ultimately, our study was conducted to inform the development of a culturally tailored DSMES program; the true test of the transferability and relevance of our findings to broader communities will be evaluated in a trial of the intervention that results from this qualitative work. The practitioners who participated in our research reported observing a wide range of cultural beliefs and practices through their interactions with AfC communities and discussed the ways they perceive these to impact on DSMES provision. However, in this analysis we have not sought to express the experiences of people living with T2D and how they perceive their culture impacts their engagement with DSMES; it is, therefore, not clear to what extent the perceptions of these practitioners reflect what AfC people living T2D themselves think.

Our study demonstrates the challenges faced by healthcare practitioners providing culturally appropriate DSMES to AfC communities. While the practitioners demonstrated a significant level of cultural sensitivity and had knowledge of a broad range of cultural beliefs and practices, they lacked confidence in their ability to support AfC groups. Overall, practitioners perceived a poor level of cultural competence within their practice and were seeking greater training and resources to support them in developing their understanding.

Acknowledgements We would like to thank the healthcare practitioners who volunteered to participate in this research.

Contributors All authors have made substantial contributions to this study. LMG, CR and SH were responsible for the conception and design of the study. LMG, $\mathrm{CR}$, SH and AM developed the protocol and study approach. LMG undertook data collection and drafted the manuscript. LMG and CR undertook data analysis. All authors read, revised and approved the final manuscript. LMG is a guarantor.
Funding This report is independent research arising from a Career Development Fellowship (LMG, CDF-2015-08-006) supported by the National Institute for Health Research. LMG and AM are supported by the National Institute for Health Research (NIHR) Applied Research Collaboration South London at King's College Hospital NHS Foundation Trust.

Disclaimer The views expressed in this publication are those of the author(s) and not necessarily those of the NHS, the National Institute for Health Research or the Department of Health.

Competing interests None declared.

Patient consent for publication Not required.

Ethics approval Ethical approval was gained from King's College London (LRS15/16-3240) and the London-Chelsea National Research Ethics Committee (16-LO1191). The study is reported according to COREQ guidelines (additional file).

Provenance and peer review Not commissioned; externally peer reviewed.

Data availability statement Data are available upon reasonable request. Deidentified participant data (interview transcripts) are available from the principal investigator (LMG; ORCHID 0000-0001-9633-8759).

Open access This is an open access article distributed in accordance with the Creative Commons Attribution Non Commercial (CC BY-NC 4.0) license, which permits others to distribute, remix, adapt, build upon this work non-commercially, and license their derivative works on different terms, provided the original work is properly cited, appropriate credit is given, any changes made indicated, and the use is non-commercial. See: http://creativecommons.org/licenses/by-nc/4.0/.

ORCID iD

Louise M Goff http://orcid.org/0000-0001-9633-8759

\section{REFERENCES}

1 Wilson C, Alam R, Latif S, et al. Patient access to healthcare services and optimisation of self-management for ethnic minority populations living with diabetes: a systematic review. Health Soc Care Community 2012;20:1-19.

2 Becker E, Boreham R, Chaudhury M, et al. Health survey for England 2004. The health of minority ethnic groups. London: Joint Health Surveys Unit, National Centre for Social Research, Department of Epidemiology and Public Health at the Royal Free and University College Medical School, 2006.

3 Tillin T, Hughes AD, Mayet J, et al. The relationship between metabolic risk factors and incident cardiovascular disease in Europeans, South Asians, and African Caribbeans: SABRE (Southall and Brent revisited) -- a prospective population-based study. J Am Coll Cardiol 2013;61:1777-86.

4 Paul SK, Owusu Adjah ES, Samanta M, et al. Comparison of body mass index at diagnosis of diabetes in a multi-ethnic population: a case-control study with matched non-diabetic controls. Diabetes Obes Metab 2017;19:1014-23.

5 Healthcare for London. Diabetes guide for London, 2009.

6 National Institute for Health and Care Excellence. Type 2 diabetes in adults: management (NG28), 2015.

7 Department of Health. National service framework for diabetes standards. London, UK: Department of Health, 2001.

8 Brown SA. Interventions to promote diabetes self-management: state of the science. Diabetes Educ 1999;25:52-61.

9 Glasgow RE, Anderson RM. In diabetes care, moving from compliance to adherence is not enough. something entirely different is needed. Diabetes Care 1999;22:2090-2.

10 Funnell MM, Brown TL, Childs BP, et al. National standards for diabetes self-management education. Diabetes Care 2008;31:S97-104.

11 Healthcare Commission. Managing diabetes: improving services for people with diabetes, 2007.

12 Brown K, Avis M, Hubbard M. Health beliefs of African-Caribbean people with type 2 diabetes: a qualitative study. Br J Gen Pract 2007:57:461-9.

13 Sarkar U, Piette JD, Gonzales R, et al. Preferences for selfmanagement support: findings from a survey of diabetes patients in safety-net health systems. Patient Educ Couns 2008;70:102-10.

14 Stone MA, Patel N, Drake L, et al. Making education in diabetes culturally appropriate for patients. Practice Nursing 2006;17:621-5.

15 Creamer J, Attridge M, Ramsden M, et al. Culturally appropriate health education for type 2 diabetes in ethnic minority groups: an updated cochrane review of randomized controlled trials. Diabet Med 2016;33:169-83. 
16 Liu J, Davidson E, Bhopal R, et al. Adapting health promotion interventions to meet the needs of ethnic minority groups: mixedmethods evidence synthesis. Health Technol Assess 2012;16:1-469.

17 Di Noia J, Furst G, Park K, et al. Designing culturally sensitive dietary interventions for African Americans: review and recommendations. Nutr Rev 2013;71:224-38.

18 O'Mara-Eves A, Brunton G, McDaid D, et al. Community engagement to reduce inequalities in health: a systematic review, meta-analysis and economic analysis. Public Health Res 2013:1:1-526.

19 Goff LM, Moore AP, Rivas C, et al. Healthy eating and active lifestyles for diabetes (HEAL-D): study protocol for the design and feasibility trial, with process evaluation, of a culturally tailored diabetes self-management programme for African-Caribbean communities. BMJ Open 2019;9:e023733.

20 Braun V, Clarke V. Using thematic analysis in psychology. Qual Res Psychol 2006;3:77-101.

21 Ziebland S, McPherson A. Making sense of qualitative data analysis: an introduction with illustrations from DIPEx (personal experiences of health and illness). Med Educ 2006;40:405-14.

22 Mayberry RM, Mili F, Ofili E. Racial and ethnic differences in access to medical care. Med Care Res Rev 2000;57:108-45.

23 Shen MJ, Peterson EB, Costas-Muñiz R, et al. The effects of race and racial concordance on patient-physician communication: a systematic review of the literature. J Racial Ethn Health Disparities 2018;5:117-40.

24 Nthangeni G, Steyn NP, Alberts M, et al. Dietary intake and barriers to dietary compliance in black type 2 diabetic patients attending primary health-care services. Public Health Nutr 2002;5:329-38.

25 Majeed-Ariss R, Jackson C, Knapp P, et al. A systematic review of research into black and ethnic minority patients' views on selfmanagement of type 2 diabetes. Health Expect 2015;18:625-42.

26 D'Eramo-Melkus G, Spollett G, Jefferson V, et al. A culturally competent intervention of education and care for black women with type 2 diabetes. Appl Nurs Res 2004;17:10-20.

27 Keyserling TC, Samuel-Hodge CD, Ammerman AS, et al. A randomized trial of an intervention to improve self-care behaviors of
African-American women with type 2 diabetes: impact on physical activity. Diabetes Care 2002;25:1576-83.

28 Rodriguez JC. Culturally competent dietetics: increasing awareness, improving care. J Am Diet Assoc 2010;110:S7.

29 Truong M, Paradies Y, Priest N. Interventions to improve cultural competency in healthcare: a systematic review of reviews. BMC Health Serv Res 2014;14:99.

30 Albright TL, Parchman M, Burge SK, et al. Predictors of self-care behavior in adults with type 2 diabetes: an RRNeST study. Fam Med 2001;33:354-60.

31 McDonald PE, Wykle ML, Misra R, et al. Predictors of social support, acceptance, health-promoting behaviors, and glycemic control in African-Americans with type 2 diabetes. $J$ Natl Black Nurses Assoc 2002;13:23-30.

32 Shaya FT, Chirikov VV, Howard D, et al. Effect of social networks intervention in type 2 diabetes: a partial randomised study. $J$ Epidemiol Community Health 2014;68:326-32.

33 Newlin K, Dyess SM, Allard E, et al. A methodological review of faithbased health promotion literature: advancing the science to expand delivery of diabetes education to black Americans. $J$ Relig Health 2012;51:1075-97.

34 Samuel-Hodge CD, Headen SW, Skelly AH, et al. Influences on dayto-day self-management of type 2 diabetes among African-American women: spirituality, the multi-caregiver role, and other social context factors. Diabetes Care 2000;23:928-33.

35 Spruill IJ, Magwood GS, Nemeth LS, et al. African Americans culturally specific approaches to the management of diabetes. Glob Qual Nurs Res 2015;2. doi:10.1177/2333393614565183. [Epub ahead of print: 21 Jan 2015].

36 de-Graft Aikins A. Healer Shopping in Africa: new evidence from rural-urban qualitative study of Ghanaian diabetes experiences. BMJ 2005;331:737.

37 de-Graft Aikins A, Unwin N, Agyemang C, et al. Tackling Africa's chronic disease burden: from the local to the global. Global Health 2010;6:5. 